\title{
Chiari Malformasyonlu Hastaların BOS Akım Parametrelerinin Normal Sağ lıklı Grup ile Kıyaslaması
}

\author{
Comparison of CSF Flow Parameters of Patients with Chiari Malformation with Healthy Individuals
}

Temel Fatih Yilmaz

Bezmialem Vakıf Üniversitesi, Tip Fakültes Radyoloji Anabilim Dalı, İstanbul, Türkiye

\section{Özet}

Chiari malformasyonu (CM) serebellar tonsillerin foramen magnumun altına 5 mm'den fazla indiği bir hastalıktır. Semptomatik olmayan Chiari hastalarının nasıl yönetileceği konusunda fikir birliği yoktur. Çalışmamızda Chiari malformasyonu olan hastalarda BOS akım parametrelerinin normal sağlıklı grup ile kıyaslayarak farklılıkları ortaya koymayı hedefledik. Ocak 2016 ile Aralık 2019 arasında faz kontrast BOS akım MRG incelemesi yapılan 85 Chiari malformasyonlu hastayı ardışı olarak değerlendirdik. 18 ile 60 yaş arasındaki bireyleri dahil ettik. Ayrıca 29 adet sağlıklı gönüllü dahil edilerek faz-kontrast BOS akım MR görüntülemesi yapıldı. İleri hacim (FV), ters hacim (RV), akuaduktal strok hacmi (ASV), ortalama alan ve tepe hızı içeren BOS akıș parametrelerini değerlendirildi. Ayrıca CM hastaların volümetrik T2 CISS görüntülemelerinden herniye tonsil ve foramen magnum çapları da kaydedildi. Akuaduktal strok volümünün CM hastalarında anlamlı olarak arttığı gözlendi ( $\mathrm{p}=0,049)$. Ortalama AS alanı CM hastalarında normal gruba oranla istatistiksel olarak anlamlı geniş bulunmuştur $(\mathrm{p}<0,001)$. Herniye tonsil ile foramen magnum çaplarının BOS akım parametreleri ile karşılaştırıldığında; herniye tonsil çapı arttıkca ASV azalmakta $(p=0.002, r=-0.333)$, foramen magnum çapı arttıkça da ASV azalmakta idi $(p=0.020, r=-0.251)$. CM hastalarında faz kontrast MR tekniği ile BOS akım parametrelerinin değerlendirmede akuaduktus Silvi düzeyi kolay ve pratik olması ve daha doğru sonuçlar vermesi nedeni ile kullanılabilecek anatomik bölgedir. CM hastalarında artmış ASV ve ortalama AS alan değerleri izlenmekte birlikte olağan dışı BOS akım değerlerini bize bildirmektedir.

Anahtar Kelimeler: BOS akım, mr, chiari malformasyonu, akuaduktal vurum hacmi, faz kontrast MR

\section{Abstract}

Chiari malformation (CM) is a disease in which cerebellar tonsils descend more than $5 \mathrm{~mm}$ below the foramen magnum. There is no consensus on how to manage non-symptomatic patients with CM. In our study, we tried to reveal the differences in CSF flow parameters in patients with Chiari malformation by comparing them with the normal healthy group. We evaluated 85 patients with Chiari malformation, who underwent phase contrast CSF flow MRI between January 2016 and December 2019, consecutively. We included individuals aged between 18 and 60 years. In addition, 29 healthy volunteers were included and phase-contrast CSF flow MR imaging was performed. CSF flow parameters including forward volume (FV), reverse volume (RV), aqueductal stroke volume (ASV), mean area, and peak velocity were evaluated. In addition, the diameters of the herniated tonsil and foramen magnum were recorded from volumetric T2 CISS images of CM patients. It was observed that the aqueductal stroke volume increased significantly in CM patients $(p=0.049)$. The mean area of AS was found to be statistically significantly larger in CM patients compared to the normal group $(p<0.001)$. When the diameters of the herniated tonsil and foramen magnum were compared with the CSF flow parameters; ASV decreased as the diameter of the herniated tonsil increased $(p=0.002, r=-0.333)$, and as the diameter of the foramen magnum increased, ASV decreased $(p=0.020, r=-0.251)$. Aqueductus Sylvii level is an anatomical location that can be used in CM patients for the evaluation of CSF flow parameters with phase contrast MR technique, since it is easy and practical and gives more accurate results. Increased ASV and average AS field values are observed in CM patients, and these are unusual CSF flow values.

Keywords: MR, aquaductal stroke volume, phase contrast MR, CSF flow

Received 23.04.2021 Accepted 17.08.2021 Online published 23.08.2021 


\section{Giriş}

Chiari malformasyonu (CM) serebellar tonsillerin foramen magnumun altına 5 mm'den fazla indiği bir hastalıktır (1-3). CMI, hastaların\% 50'sinden fazlasında siringomiyeli ile ilişkilidir (4-5). Hipoplastik posterior fossa ve kranioservikal bileşkede stenozun neden olduğu düşünülen CMI ile ilişkili siringomiyelinin karakteristik nörolojik ve radyolojik özellikleri olmasına rağmen, kesin mekanizmas1 bilinmemektedir (6). Semptomatik olmayan Chiari hastalarının nasıl yönetileceği konusunda fikir birliği yoktur. Tedavi kararı vermede hasta kliniğinin yanı sira posterior fossa ve kranioservikal bileşke düzeyine yönelik magnetik rezonans (MR) görüntülemesi faydalı olabilmektedir. Faz kontrast MR görüntüleme (PC-MRI) ile $\mathrm{CM}$ olan hastalarda BOS akım dinamikleri ile alakalı değerli bilgiler elde edilebilmektedir (7). Çalışmamızda Chiari malformasyonu olan hastalarda BOS akım parametrelerinin normal sağlıklı grup ile kıyaslayarak farklılıkları ortaya koymayı hedefledik.

\section{Yöntem ve Gereçer}

\section{Hasta seçimi}

Ocak 2016 ile Aralık 2019 arasında faz kontrast BOS akım MRG incelemesi yapılan 85 Chiari malformasyonlu hastayı ardışık olarak değerlendirdik. 18 ile 60 yaş arasındaki bireyleri dahil ettik. $5 \mathrm{~mm}$ 'den fazla tonsil ektopisi olan hastalar dahil edildi. Operasyon sonrası yapılan çekimler dışlandı. Çalışmamıza ayrıca 29 adet sağlıklı gönüllü dahil edilerek faz-kontrast BOS akım MR görüntülemesi yapıldı. Hastaları chiari $(\mathrm{n}=$ $85)$ ve normal $(n=29)$ olmak üzere iki gruba ayırdık. İleri hacim (FV), ters hacim (RV), akuaduktal strok hacmi (ASV), ortalama alan ve tepe hızı içeren BOS akış parametrelerini değerlendirdik. ASV, FV ve RV toplamının yarısı olarak hesaplandı. Çalışmamıza Bezmialem Vakıf Üniversitesi etik komitesi tarafından onay verilmiştir.

\section{Görüntüleme Tekniği}

1.5 Tesla MR cihazı ile (Avanto; Siemens Medical Solution, Erlangen, Almanya) kafa sarmalı kullanılarak MR yapıldı. BOS akışının kantitatif değerlendirmesi, iki boyutlu (2D) Q FLOW faz-kontrast MR anjiyografi tekniği ile eksenel düzlemde elde edilen görüntülerle gerçekleștirildi. Akuaduktus Silvinin (AS) en geniş yeri olan orta üçte birlik kısımdan axial planda görüntüler elde edildi. Her hasta için PC-MRI süresi yaklaşık 5 dakikaydı. İlk olarak orta hat sagital, koronal ve aksiyal T1 W ön görüntüler elde edildi. "Mean modulus," "magnitude of complex difference" ve"directional phase difference" görüntülemeler semiaksiyel planda AS ye dik olacak şekilde elde edildi. Aksiyel düzlemde görüntüler için, kalp hızına göre 14-30 kardiyak faz kesiti için kullanılan parametreler: TR: $31,25 \mathrm{~ms}$, TE: $8,06 \mathrm{~ms}$, kesit kalınlığı: 3 mm, NSA: 1, FOV: $16 \times 10$ $\mathrm{cm}$, matris $128 \times 256$ ve flip angle $10^{\circ}$. Kardiyak tetikleme, MR uyumlu elektrotlarla (Kendall, Arbo, Tyco International, Neustadt, Almanya) geriye dönük olarak gerçekleştirildi. Akış hassasiyeti (venc) $20 \mathrm{~cm}$ / s olarak belirlendi. Kaudokraniyal yöndeki akım (diyastolik veya ters akış) negatif olarak tanımlanırken kraniokaudal yöndeki akış (sistolik veya ileri akış) pozitif olarak belirlendi. PC-MRI dan sonra sagital T2 CISS ve T2 SPACE sekanslar1 da elde edildi. 3DT2 CISS için kullanılan parametreler şunlardı: kesit kalınlığı: $1 \mathrm{~mm}$, FOV: $200 \mathrm{~mm}$, matris $290 \times 320$, TR: $6,06 \mathrm{~ms}$, TR: $2,61 \mathrm{~ms}$ ve flip angle $70^{\circ}$. 3D-T2 SPACE için kullanılan parametreler şunlardı: kesit kalınlı̆̆ı: $1 \mathrm{~mm}$, FOV: $240 \mathrm{~mm}$, matris $231 \times 256$, TR: 2500 ve TR: 501.

\section{MR analizi}

Tüm hastalardan ve kontrol grubundan elde edilen görüntüler Siemens kullanıcı konsolu (Argus yazılımı, Siemens, Erlangen, Almanya) kullanılarak değerlendirildi. BOS akış ölçümü için 'PC-MRI aksiyel through plane' görüntüler kullanıldı. Aksiyel görüntüde AS en geniş yerinden ölçümler yapılmıştır. Akış konturları 'Region of Interest (ROI)' tarafindan çizildi (Şekil 1) ve ROI, bir kardiyak döngü sırasında elde edilen aksiyel faz görüntülerine tamamen kopyalandi. Tepe ve ortalama hiz $(\mathrm{cm} / \mathrm{s})$, ileri ve geri akış hacmi $(\mu 1)$, net ileri akış hacmi $(\mu 1)$, ASV (aqueductal strok hacmi) ( $\mu 1)$, AS 
alanı (mm2) hesaplandı (Şekil 2). Ayrıca CM foramen magnum çapları da kaydedildi (Şekil hastaların volümetrik T2 CISS 3).

görüntülemelerinden herniye tonsil ve

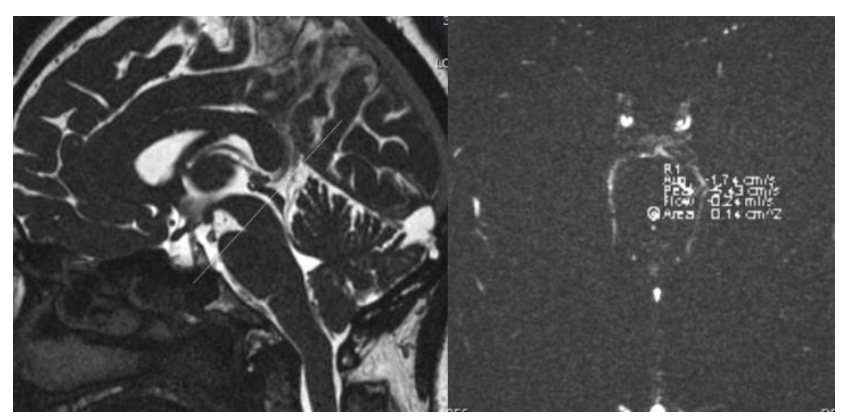

Şekil 1. Ampulla'ya dik olarak alınan aksiyel görüntüler ve tüm akuaduktus'u içerecek şekilde ROİ'nin yerleştirilmesi

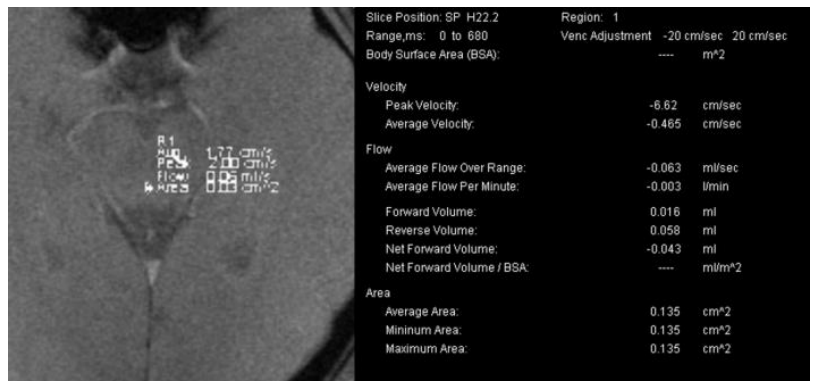

Şekil 2. Sylvian akuaduktus tan elde edilen BOS akış parametrelerinin özet tablosu.

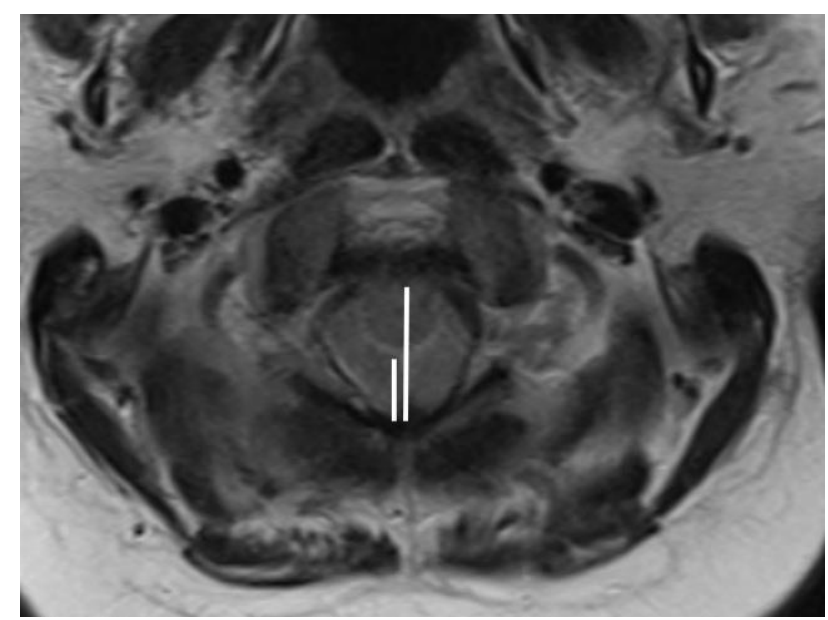

Şekil 3. Aksiyel T2 ağırlıklı görüntülemede herniye tonsil ve foramen magnum çaplarının ölçümü 
Tablo 1. İstatistiksel veriler

\begin{tabular}{|c|c|c|c|c|c|c|c|}
\hline & $\mathbf{n}$ & $\begin{array}{c}\text { PPS/AS } \\
(\text { mean } \pm \text { SD })\end{array}$ & $\begin{array}{c}\text { ASV } \\
(\text { mean } \pm \text { SD })\end{array}$ & $\begin{array}{c}\text { Ortalama } \\
\text { alan } \\
(\text { mean } \pm \text { SD })\end{array}$ & $\begin{array}{l}\text { İleri Volüm } \\
(\text { mean } \pm \text { SD) }\end{array}$ & $\begin{array}{l}\text { Geri Volüm } \\
(\text { mean } \pm \text { SD) }\end{array}$ & $\begin{array}{l}\text { Pik akım hız } \\
(\text { mean } \pm \text { SD })\end{array}$ \\
\hline $\begin{array}{l}\mathrm{CM} \\
\text { hastaları }\end{array}$ & 85 & $2.30 \pm 0.62$ & $31.24 \pm 27.17$ & $0,08 \pm 0.05$ & $23,32 \pm 25,07$ & $39,15 \pm 33,70$ & $5,00 \pm 2,44$ \\
\hline $\begin{array}{l}\text { Normal } \\
\text { grup }\end{array}$ & 29 & $4.12 \pm 0.86$ & $23.88 \pm 12.14$ & $0,04 \pm 0.02$ & $17,79 \pm 10,58$ & $30,28 \pm 14,63$ & $5,02 \pm 1,62$ \\
\hline$P$ değeri & & 0,001 & 0,049 & 0,004 & 0.102 & 0.054 & 0.949 \\
\hline
\end{tabular}

CM: Chiari malformasyonu

\section{Istatistiksel analiz}

Tüm istatistiksel analizler SPSS Windows sürüm 26.0 yazılım paketi (IBM Corp., New York, NY; eski adıyla SPSS Inc., Chicago, IL) kullanılarak yapıldı. CM olguları ile normal grup karşılaştırıldı. İlk olarak değişkenlerin iki gruptan dağılımı Shapiro Wilk normallik testi kullanılarak değerlendirildi. Değişkenlerin iki farklı grup arasında dağılımı normal olduğundan grup karşılaştırması için bağımsız t testi kullanıldı. $P<0,05$ değeri istatistiksel olarak anlamlı kabul edildi. Herniye tonsil ve foramen magnum çapları ile BOS akım parametreleri arasındaki ilişki Spearman korelasyon testi kullanılarak yapıldı.

\section{Sonuçlar}

CM hastaların yaş ortalaması $34.15 \pm 16.84$, normal grubun ise $36.14 \pm 11.37$ idi. İleri ve ters BOS akımının ortalamasını temsil eden akuaduktal strok volümünün $\mathrm{CM}$ hastalarında anlamlı olarak arttığ gözlendi $(\mathrm{p}=0,049)$. Ortalama AS alanı CM hastalarında normal gruba oranla istatistiksel olarak anlamlı geniş bulunmuştur ( $\mathrm{p}<0,001$ ). Pik hız, ileri volüm, geri volüm açısından istatistiksel olarak anlamlı bir fark yoktur. CM hastalarının kendi içlerinde herniye tonsil çapı ile foramen magnum çaplarının BOS akım parametreleri ile karşılaştırıldığında herniye tonsil çapı arttıça ASV azalmakta ( $\mathrm{p}=0.002, \mathrm{r}=$ 0.333 ), foramen magnum çap1 arttıkça da ASV azalmakta idi $(\mathrm{p}=0.020, \mathrm{r}=-0.251)$.

\section{Tartışma}

$\mathrm{CM}$ olan hastalarda günümüze kadar yapılmış birçok çalışma bulunmaktadır. Krueger ve arkadaşları CM hastalarında, semptomatik olan ve olmayan hastalarda pik hız araştırması yapmış olup bunu foramen magnum seviyesinde ölçmüşlerdir çalışmalarında semptomatik durumun foramen magnum düzeyinde hızlarda değişikliğe neden olmadığını göstermişlerdir (8). Biz çalışmamızda normal hasta grubu ile $\mathrm{CM}$ hastaları arasında farklılık gösteremedik. $\mathrm{Bu}$ konuda ayrıca McGirt ve arkadaşları, baş ağrısı ile BOS akım parametreleri arasındaki iliş̧kiyi araştıran yazılarında, oksipital baş ağrısının BOS akım dinamiklerindeki bozulma ile daha yakından ilişkili olduğunu ortaya koymuşlar (9) .Biz çalışmamızda, CM hastalarının normal gruba göre BOS akım dinamiklerinde oluşturduğu farklılıkları araştırdık ve semptomatik duruma göre ayrım yapmadik.

Koç ve arkadaşları ise siringomyeli ile prezente tip $1 \mathrm{CM}$ hastalarında foramen magnum ve sirinks kavitesinden faz kontrast MR incelemesi yaparak operasyon öncesi ve sonras1 BOS akım dinamiklerinin karşılaş̧ırmışlar. Operasyon sonrası BOS akımındaki heterojen paternin \%61 oranda sinüzoidal paterne döndüğünü bildirmişlerdir (3). Bizim çalışmamızda amaç operasyon öncesi yapılan inceleme ile BOS akım parametrelerindeki normal dişı değişimleri göstererek operasyona 1 şı tutmaktır. BOS akım analizini rutin görüntüleme olan akuaduktus silvi seviyesinden yapmamızın nedeni günlük pratikte $\mathrm{CM}$ olan hastaların olağandışı bulgularını ortaya koymaktır.

Subaraknoid boşluklarda, özellikle foramen magnum seviyesinde BOS parametrelerinin ölçümleri zor olup hata ve artefaktlara neden olabilir (10-12). ASV ölçümü akuaduktus silvi düzeyinde daha kolay ve daha pratiktir. Özellikle kemik komşuluğu olmaması, sıvı yumuşak doku geçişinin MR da artmış uzaysal rezolüsyon bağlı daha iyi yapılması, akuaduktus silvinin foramen magnum ve 
servikal düzeye göre daha kolay değerlendirebilir olması ve yuvarlak şekli ile daha kolay ROI yerleştirilebilmesi nedeni ile BOS akımı hakkında daha doğru bilgi sağlar. $\mathrm{Bu}$ nedenle, foramen magnum seviyesinde ve üst servikal seviyede hız ölçümü yapılmadı.

AS alanının CM olan hastalarda yüksek olmasinı lateral medüller sisternde tonsiller ektopiye sekonder daralma ve buna bağlı BOS akışındaki zorlanmaya bağladık. ASV değerinin CM hastalarındaki yüksekliği ortalama AS alanındaki artışa ve foramen magnum seviyesindeki BOS klirensindeki azalmaya bağladik. Foramen magnum ve herniye tonsil çapı ile ASV arasındaki negatif korelasyon da hipotezimizi desteklemekte akuaduktus Silvi düzeyindeki BOS akım değerlendirmesinin önemini vurgulamaktadır.

Çalışmamıza ait birtakım kısıtlamalar mevcuttur. Bunlardan ilki çalışmamızın retrospektif ve tek merkezli olmasıdır. İkincisi

\section{KAYNAKLAR}

1. Barkovich AJ, Wippold FJ, Sherman JL, Citrin CM. Significance of cerebellar tonsillar position on MR. AJNR Am J Neuroradiology 1986;7:795799.

2. Smith BW, Strahle J, Bapuraj JR, Muraszko KM, Garton HJ, Maher CO. Distribution of cerebellar tonsil position: implications for understanding Chiari malformation. J Neurosurg 2013;119:812819.

3. Koc K, Anik Y, Anik I, Cabuk B, Ceylan S. Chiari malformation with syringomyelia: correlation phase-contrast cine MR imaging and outcome. Turk Neurosurg 2007;17:183-192.

4. Wang CS, Wang X, Fu CH, Wei LQ, Zhou DQ, Lin JK. Analysis of cerebrospinal fluid flow dynamics and morphology in Chiari I malformation with cine phase-contrast magnetic resonance imaging. Acta Neurochir 2014;156:707713.

5. Lloyd RA, Fletcher DF, Clarke EC, Bilston LE. Chiari malformation may increase perivascular cerebrospinal fluid flow into the spinal cord: A subject-specific computational modelling study. $J$ Biomech 201;65:185-193.

6. Levine DN. The pathogenesis of syringomyelia associated with lesions at the foramen magnum: a critical review of existing theories and proposal of new hypothesis. $J$ Neurol Sci 2004;220:3-21.

7. Panigrahi M, Reddy BP, Reddy AK, Reddy JJ. CSF flow study in Chiari I malformation. Childs Nerv Syst. 2004;20:336-40. dekompresyon cerrahisi öncesinde ve sonrasında aquaduktus silvi düzeyinden BOS akım parametrelerinin ölçülmemesi ve ayrıca postoperatif takiplerinin yapılmamış olmasıdır. Üçüncüsü CM hastalarına tiplendirme yapılıp birbirleri ile kıyaslama yapılmamış olmasıdır.

\section{Sonuc}

CM hastalarında faz kontrast MR tekniği ile BOS akım parametrelerinin değerlendirmede akuaduktus Silvi, kolay ve pratik olması ile daha az artefakta bağlı daha doğru sonuçlar verebilecek olması nedeni ile kullanılabilecek anatomik bölgedir. CM hastalarında artmış ASV ve ortalama AS alan değerleri izlenmekte birlikte olağan dışı BOS akım değerlerini bize bildirmektedir. $\mathrm{Bu}$ değerlerin gelecek çalışmalar ışığında operasyon öncesi kullanılabilecek parametreler olabileceğini düşünmekteyiz.
8. Krueger KD, Haughton VM, Hetzel S. Peak CSF velocities in patients with symptomatic and asymptomatic Chiari I malformation. AJNR Am J Neuroradiol. 2010;31:1837-41.

9. McGirt MJ, Nimjee SM, Floyd J, Bulsara KR, George TM. Correlation of cerebrospinal fluid flow dynamics and headache in Chiari I malformation. Neurosurgery. 2005;56:716-21; discussion 716-21.

10. Schaffer N, Martin B, Loth F. Cerebrospinal fluid hydrodynamics in type I Chiari malformation. Neurol Res 2011;33:247-260.

11. Shah S, Haughton V, Munoz del Rio A. CSF flow through the upper cervical canal in Chiari I malformation. AJNR Am J Neuroradiology 2011;32:1149-1153.

12. Wentland AL, Wieben O, Korosec FR, et al. Accuracy and reproduccility of phase-contrast MR imaging measurements for CSF flow. $\mathrm{Am} J$ Neuroradiol 2010;31:1331-1336. 